\title{
Optimization of Antioxidant Properties of Creams with Berry Extracts by Artificial Neural Networks
}

\author{
K. Makarova*, K. Zawada, D. Wagner and J. Skowyra \\ Department of Physical Chemistry, Faculty of Pharmacy, The Medical University of Warsaw, \\ S. Banacha 1, 02-097 Warsaw, Poland
}

\begin{abstract}
Oxidative stress and the excess of free radicals accelerate the ageing process of human skin. The application of skin cream with antioxidant compounds could reduce the damage caused by free radicals. In this work we studied two types of skin creams with extracts from aronia (Aronia melanocarpa), elderberry (Sambucus nigra) and bilberry (Vaccinium myrtillus) because of their high content of anthocyanins, i.e. strong natural antioxidants. The 2,2diphenyl-1-picrylhydrazyl (DPPH) radical scavenging ability of the skin creams with berry extracts were studied with ESR spectroscopy. The artificial neural networks were applied to optimize the berry extract concentration and storage time for oil-in-water and water-in-oil creams. Based on experimental results chokeberry and elderberry extracts in oil-in-water cream base revealed higher DPPH radical scavenging ability than in the corresponding water-in-oil. Artificial neural networks predicts maxima of DPPH radical scavenging for 1-week stored elderberry $(2.23 \mathrm{mg} \mathrm{DPPH} / \mathrm{g})$ and 1-week stored chokeberry (5.84 mg DPPH/g) and bilberry (5.26 mg DPPH/g) $0.76 \%$ extracts in oil-in-water creams. The maxima of DPPH radical scavenging for water-in-oil creams were predicted for 6 -week stored $0.8 \%$ aronia extract, freshly prepared $0.76 \%$ bilberry extract and 1 -week stored $0.56 \%$ elderberry extract. The artificial neural networks predicted values are in good agreement with the experimental values. DPPH-EPR could be combined with artificial neural networks to optimize the extract concentration, and the type of cream base as well as to predict the effect of storage based on a limited number of experiments and samples.
\end{abstract}

DOI: 10.12693/APhysPolA.132.44

PACS/topics: 07.05.Mh, 33.35.+r, 61.72.Hh, 76.30.-v, 87.64.kh, 87.80.Lg

\section{Introduction}

Normal skin ageing is characterized by thinning of the epidermis, resulting in fine wrinkles, dry and thin appearance, hyperpigmentation etc. [1]. Such extrinsic factors as photo-oxidative stress and reactive oxygen species (ROS) induced collagen degradation were proposed as possible mechanisms of skin ageing $[2,3]$. Correspondingly, an increased exposure to environmental oxidative stress factors as well as reduced overall antioxidant defense of the skin may accelerate the skin ageing process. Thus, topical application of antioxidants may be beneficial for protecting the skin against environmental factors causing oxidative stress and the age-related increase of oxidative stress [4]. Currently antioxidants are widely incorporated into a variety of anti-ageing skin care systems, such as creams, lotions etc. The commonly used antioxidant ingredients include vitamins $\mathrm{A}, \mathrm{C}$, and $\mathrm{E}$ as well as plant polyphenols such as tannins and flavonoids [5-7].

Recently, anthocyanins, a subcategory of the flavonoids class, have received increased attention due to their significant antioxidant, anti-inflammatory and antimutagenic effects [8]. The strong correlation between anthocyanin content and antioxidant capacity was also reported $[9,10]$.

Berry fruits are generally rich in anthocyanins. It was shown in a number of studies that supplementation

*corresponding author; e-mail: kmakarova@wum.edu.pl with anthocyanin-rich berries was effective in reducing oxidative stress associated with ageing, and were beneficial in reversing the age-related neuronal and behavioral changes [11]. It was also reported that bilberries (Vaccinium myrtillus L.), elderberries (Sambucus nigra) and aronia (chokeberry) (Aronia Melanocarpa) have high antioxidant properties $[12,13]$. The anthocyanins concentration for bilberry, elderberry, and aronia were reported to be within the range of $300-698 \mathrm{mg} / 100 \mathrm{~g}$ fresh weight [14-17]. The antioxidant activity of bilberry, elderberry and aronia were extensively investigated by oxygen radical absorbance capacity (ORAC) $[15,18]$ and other methods, including DPPH assay [19-25]. The studies showed high activity of all those fruits in all performed tests, but the highest antioxidant activity was obtained for aronia.

Owing to their high antioxidant potential the bilberry, elderberry and aronia berries are good candidates for skin cream components. In this work we investigated the antioxidant properties of two types of cream bases (oil-inwater and water-in-oil emulsion) with berry extracts. We applied the DPPH radical assay with EPR spectroscopy. It was earlier successfully applied for the determination of radical scavenging activity of various types of food and chemical samples [26-30]. It was also used to examine the radical scavenging properties of UV-protecting creams [31]. The application of EPR spectroscopy is particularly beneficial in this case because of the sample physical properties, i.e. it being an emulsion. In the case of emulsion the opacity of the sample makes direct measurement of the antioxidant properties by UV-vis or fluorescence spectroscopy impossible. 
The experimental results (DPPH-EPR test) were supported by artificial neural network (ANN) modeling. The ANN allowed us to reduce the number of required experiments for the determination of the system parameters for a maximum of DPPH radical scavenging. In addition, the results obtained from ANN allowed us to study the possible interactions between berry extracts, cream base components, and DPPH radicals. This information is potentially important for optimizing the cream composition.

\section{Materials and methods}

\subsection{Chemicals}

All chemicals were purchased from POCh (Polish Chemical Reagents), Sigma Aldrich and Merck \& Co. The chemicals were of laboratory reagent grade and were used without further purification. Distilled water was used in all the experiments. Olive oil was bought in the local store, the same olive oil was used for the preparation of both cream bases. Elderberry powder (standardized for $10 \%$ of anthocyanins by dry weight) was obtained from Artemis Nutritionals Europe AG, and aronia extract (standardized for $15 \%$ of anthocyanins by dry weight) and bilberry extract (standardized for 25\% of anthocyanins by dry weight) from Kaden Biochemicals $\mathrm{GmbH}$.

\subsection{Preparation of cream bases and creams}

For the water-in-oil cream base (w/o) the oil phase was prepared by melting white beeswax $(15 \% \mathrm{w} / \mathrm{w})$, liquid paraffin $(25 \% \mathrm{w} / \mathrm{w})$, olive oil $(25 \% \mathrm{w} / \mathrm{w})$, cholesterol $(2.5 \% \mathrm{w} / \mathrm{w})$ and glycerin $(8 \% \mathrm{w} / \mathrm{w})$ in water bath. The water phase was prepared by dissolving borax $(1 \% \mathrm{w} / \mathrm{w})$ in distilled water. For the oil-in-water cream base $(\mathrm{o} / \mathrm{w})$ the oil phase was prepared by melting stearic acid $(10 \%$ $\mathrm{w} / \mathrm{w})$, liquid paraffin $(20 \% \mathrm{w} / \mathrm{w})$, olive oil $(20 \% \mathrm{w} / \mathrm{w})$, cetaceum $(5 \% \mathrm{w} / \mathrm{w})$, lanolin $(3 \% \mathrm{w} / \mathrm{w})$, cetyl alcohol $(1 \%$ $\mathrm{w} / \mathrm{w})$ and glycerin $(10 \% \mathrm{w} / \mathrm{w})$ in water bath. The water phase was prepared by dissolving triethanolamine $(1 \%$ $\mathrm{w} / \mathrm{w})$ in water. The water phase was gradually added to the oil phase and stirred at room temperature till a homogeneous emulsion was obtained. The berry extracts were dissolved in acidified water: ethanol 1:1 (pH 2.5), in concentrations $0.8 \% \mathrm{w} / \mathrm{w}, 0.4 \% \mathrm{w} / \mathrm{w}$ and $0.2 \% \mathrm{w} / \mathrm{w} .250 \mu \mathrm{l}$ of a berry extract solution was added to $2.5 \mathrm{~g}$ of each base type. The accelerated ageing of creams and cream bases was performed at $50^{\circ} \mathrm{C}$, in darkness, for 6 weeks, in closed vials.

\subsection{DPPH radical scavenging assay}

The antioxidant activity of the berry extracts, cream bases and creams were studied using DPPH assay. The DPPH ethanolic solution $(1.3 \mathrm{mM})$ was prepared one day before each measurement and its stability was checked prior to experiment. $20 \mu \mathrm{l}$ of the extract solution was added to the DPPH ethanolic solution. The mixture was incubated in darkness at room temperature for $45 \mathrm{~min}$, then the EPR measurements were made. Before measurement, $300 \mu \mathrm{l}$ of the acetone-water mixture $(1: 1 \mathrm{v} / \mathrm{v})$ was added to $0.15 \mathrm{~g}$ of $\mathrm{w} / \mathrm{o}$ cream and $\mathrm{w} / \mathrm{o}$ cream base and $300 \mu \mathrm{l}$ of the ethanol-water mixture $(1: 1 \mathrm{v} / \mathrm{v})$ was added to $0.15 \mathrm{~g}$ of $\mathrm{o} / \mathrm{w}$ cream and $\mathrm{o} / \mathrm{w}$ cream base. Then, the DPPH ethanolic solution $(1.3 \mathrm{mM})$ was added to each sample. The mixture was incubated in darkness at room temperature for $60 \mathrm{~min}$, then the EPR measurements were made.

The DPPH solution volume was varied both in the case of berry extracts solutions and creams to obtain the 15$80 \%$ reduction of initial DPPH concentration. For each sample the blank sample was prepared, where the sample was replaced by the solvent mixture. The concentration of DPPH was assessed by comparing the signal intensity of the sample with the signal intensity of the DPPH solution of known concentration [mg/L]. The results were calculated as milligrams of DPPH radical scavenged by $1 \mathrm{~mL}$ of berry extract solution or $1 \mathrm{~g}$ of a cream using the following equation:

$$
m_{\mathrm{DPPH}}=\frac{\left(\frac{I_{\text {blank }}-I_{\text {sample }}}{I_{\text {sample }}}\right) V_{\mathrm{DPPH}} c_{0 \mathrm{DPPH}}}{V_{\text {solution of a cream }} c_{\text {solution of a cream }}},
$$

where $m_{\mathrm{DPPH}}[\mathrm{mg}$ per $1 \mathrm{~g}$ of a cream] - mass of DPPH radical that reacted with $1 \mathrm{~g}$ of a cream, $I_{\text {blank }}$ - signal intensity of a blank, $I_{\text {sample }}$ - signal intensity of a sample, $V_{\mathrm{DPPH}}$ - the volume of $\mathrm{DPPH}$ stock solution taken for the measurement $[\mathrm{ml}], c_{0 \mathrm{DPPH}}$ - the concentration of stock solution of DPPH radical $[\mathrm{mg} / \mathrm{ml}]$,

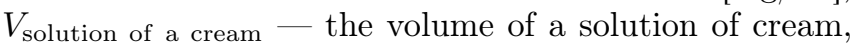
prepared by diluting the cream with a proper solvent (depending on the cream base) $[\mathrm{ml}], c_{\text {solution of a cream }-}$ concentration of a solution of a cream, prepared by diluting the cream with a proper solvent (depending on the cream base) $[\mathrm{g} / \mathrm{ml}]$.

All calculations were made for blank samples prepared with the same dilution of a stock DPPH solution as the dilution which was the consequence of adding a solution of the cream to the stock solution of DPPH radical.

\subsection{EPR measurements}

EPR measurements were performed on an X band (9.5 $\mathrm{GHz}$ ) MiniScope200 spectrometer (Magnettech), using the following parameters: central field $334.3 \mathrm{mT}$, sweep $7.1 \mathrm{mT}$, sweep time $20 \mathrm{~s}$, modulation amplitude $0.1 \mathrm{mT}$, microwave power $20 \mathrm{~mW}$. The DPPH signal was checked not to be saturated. All experiments were performed at room temperature, in triplicate.

\subsection{Artificial neural network}

\subsubsection{Artificial neural network architecture}

Matlab Neural Network Toolbox was used to design a multi layer perceptron (MLP) with the "newp" routine. The general architecture for the MLP network is shown in Fig. 1. The designed MLP network has an input layer, two hidden layers with a sigmoidal activation function and a linear output layer. The input layer I 
is a vector that consists of 3 experimental parameters of the studied system, namely concentration of the extract, time of storage in weeks, and cream base number. The single output node corresponds to DPPH scavenging ability. The number of nodes in two hidden layers are adjusted experimentally. For the training of MLP the back propagation algorithm was used. During the training, the output computed by MLP was compared with the desired output from the training set, then the difference between the outcomes (error) was calculated. Subsequently, the weights of every connection between the layers were adjusted in order to minimize the error.

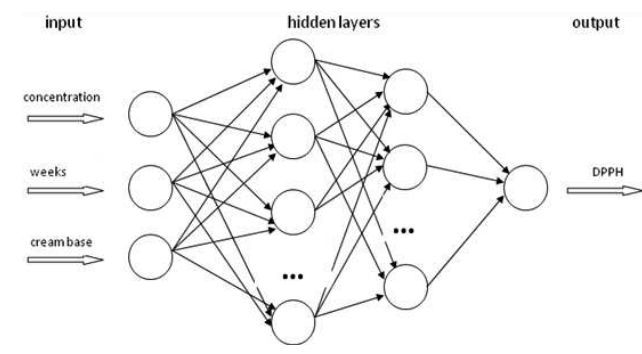

Fig. 1. MLP neural network architecture. The inputs are the concentration of the berry extract $(0-0.8 \%)$, time of storage (0-6 weeks) and the type of cream base $(\mathrm{w} / \mathrm{o}(1)$ or $\mathrm{o} / \mathrm{w}(2))$. The number of neurons in the hidden layers depends on the studied system. The output of MLP is DPPH scavenging activity.

\subsubsection{Training set construction}

The experimental data from Table I was used for construction of the training and testing sets. Two types of training sets were constructed. The first one is the extract-dependent training set which contains experimental data for one type of extract in all cream bases. The second one is the individual training set which consists of experimental data for one type of extract in one cream base. To improve the performance and robustness of the neural network all parameters in the training, testing and experimental sets were normalized by scaling their values in the range $[-1,1]$ before introduction to the network.

\section{Results and discussion \\ 3.1. DPPH radical scavenging activity}

DPPH scavenging activity of the berry extract as well as two cream bases (i.e. water-in-oil and oil-in-water) and creams with berry extracts were assessed immediately after preparation and after 3 and 6 weeks of storage.

\subsubsection{Berry extracts}

DPPH radical scavenging activity of all berry extracts increases with the extract concentration (Fig. 2). The highest value for the scavenging activity was obtained for the extract from chokeberry in each used concentration followed by bilberry and elderberry extracts. In our case the DPPH radical scavenging activity was not correlated with the anthocyanin content of the samples.
TABLE I

The testing set for neural networks. Predicted values of MLP 1 (individual) and MLP 2 (all).

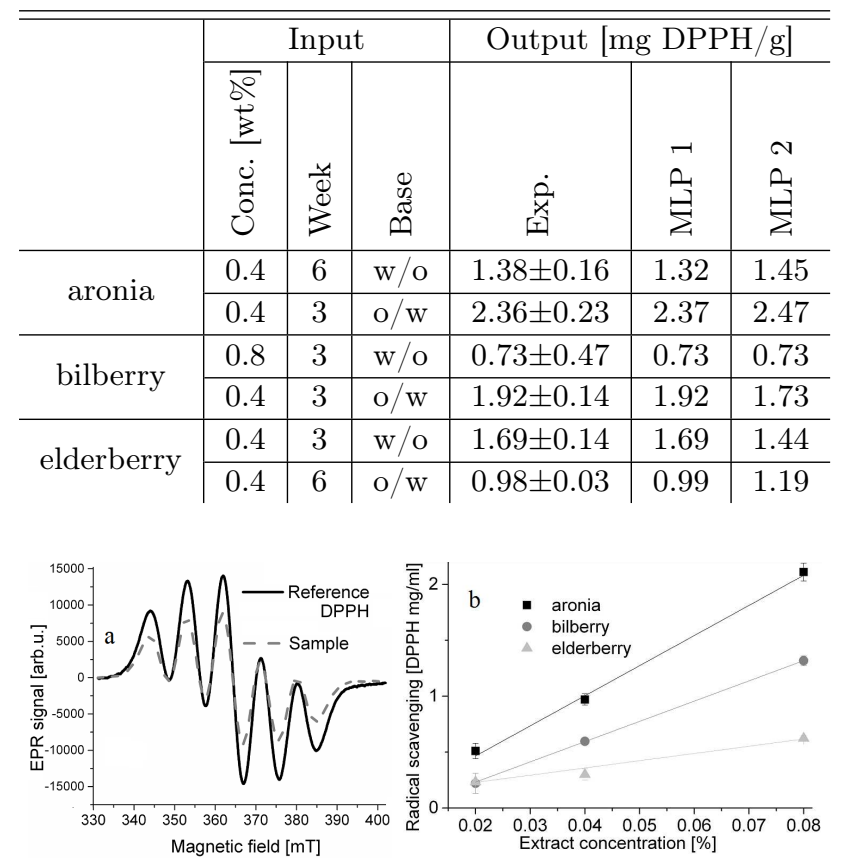

Fig. 2. (a) DPPH signal from the reference sample (ethanolic solution $1.3 \mathrm{mM}$ ) and sample with the berry extract, (b) the relation between DPPH scavenging of aronia, bilberry, and elderberry extracts and their concentration.

\subsubsection{Cream bases}

Water-in-oil cream base (w/o) shows higher antioxidant activity $(2.5 \mathrm{mg} \mathrm{DPPH} / \mathrm{g})$ than oil-in-water cream base $(\mathrm{o} / \mathrm{w})$ (1.28 mg DPPH/g) (Fig. 3). The antioxidant activity of both cream bases is most probably due to the olive oil components. Such olive oil components as phenolic compounds are well known natural antioxidants. Previously, it was shown that the radical scavenging properties of olive oil was about $1.04 \mathrm{mg} \mathrm{DPPH} / \mathrm{ml} \mathrm{[32].}$ Since the content of olive oil in both cream bases is very similar, the difference in DPPH radical scavenging ability is most likely due to the structure of cream bases. In contrast to $\mathrm{w} / \mathrm{o}$ base, in $\mathrm{o} / \mathrm{w}$ base olive oil is present in the inner phase, which could inhibit the interactions with DPPH radicals. This results in lower DPPH radical scavenging. The radical scavenging properties of both cream bases decrease with time, probably due to the thermal oxidative degradation of olive oil. However, after 6 weeks of ageing the $\mathrm{o} / \mathrm{w}$ cream base exhibited a slight increase in DPPH radical scavenging properties (1.12 mg DPPH/g) as compared with 3 weeks measurements $(0.66 \mathrm{mg} \mathrm{DPPH} / \mathrm{g})$. That could be an indication of the changes in cream base structure leading to better interaction of olive oil components with DPPH radicals, or the formation of oxidation products with better antioxidant properties. 


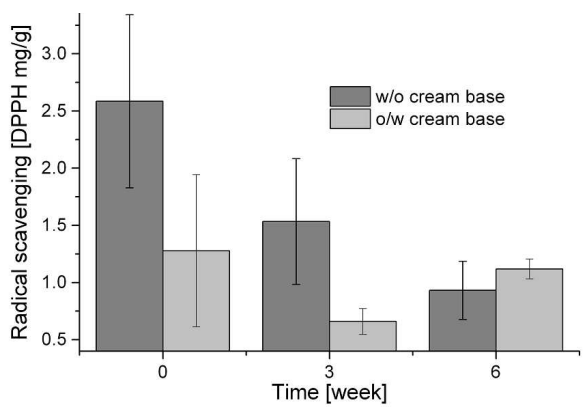

Fig. 3. DPPH scavenging of water-in-oil and oil-inwater cream bases.

\subsubsection{Creams with berry extracts - water-in-oil cream base}

Generally, the addition of different berry extracts decreases the antioxidant activity as compared with pure w/o cream base (Fig. 4). Berry extracts could accelerate the oxidation of cream base compounds, for example the olive oil. This effect is illustrated clearly in the case of elderberry extract, where the increase of the extract concentration leads to a decrease of DPPH radical scavenging properties of the cream. At the same time, the ethanol which was used as a solvent for berry extracts could partly destabilize the cream structure, as it can increase the effective hydrophile-lipophile balance (HLB) value of emulsifiers [33]. As a result, anthocyanins from berry extracts have better accessibility to DPPH radicals. This leads to an increase of the DPPH radical scavenging activity of the w/o cream samples with the extract concentration (Fig. 4, the samples with aronia and bilberry extracts).

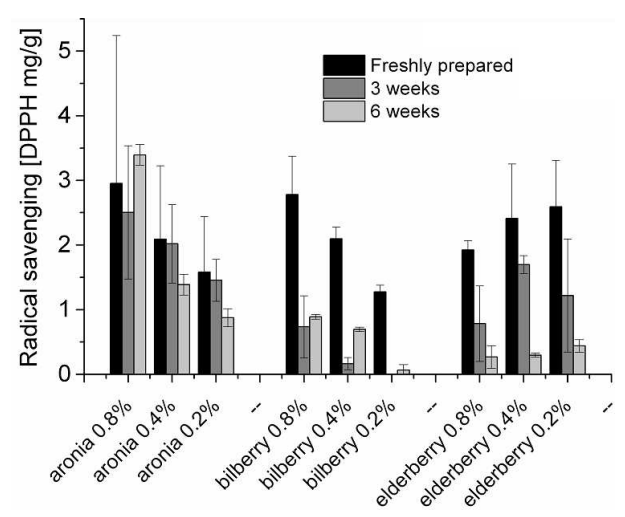

Fig. 4. DPPH radical scavenging of creams with aronia, bilberry, and elderberry extracts — water-in-oil base.

It can be suggested that there are two processes in competition: the general decrease of radical scavenging activity due to the oxidation of olive oil, and the increase of radical scavenging activity related to an increased effective concentration of the extracts. The latter would be due to the changes in cream structure, i.e. better accessi- bility of extract components to DPPH radicals. The first process dominates in freshly prepared samples, whereas the importance of the second process increases with time.

Thus, the DPPH radical scavenging activity in $\mathrm{w} / \mathrm{O}$ cream with the selected berry extracts is determined by the combined action of those two processes.

\subsubsection{Creams with berry extracts - oil-in-water cream} base

In the case of $\mathrm{o} / \mathrm{w}$ base, both extracts and DPPH radicals are located in the outer phase of the emulsion. In this case, the components of the berry extract have better accessibility to DPPH radicals. As a result, creams with the addition of berry extracts exhibit higher DPPH radical scavenging activity than those made of pure $\mathrm{o} / \mathrm{w}$ cream base and pure extracts solutions (Fig. 5).

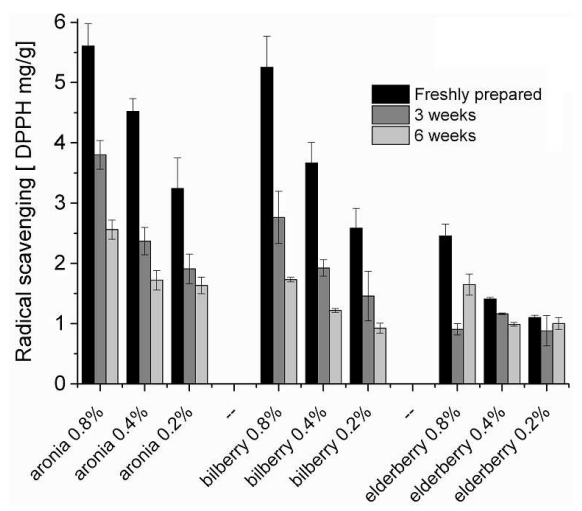

Fig. 5. DPPH radical scavenging of creams with aronia, bilberry, and elderberry extracts - oil-in-water base.

The results suggest that DPPH radical scavenging by cream base components and berry extracts is the predominating process here. However, the process of olive oil oxidation (due to the interactions with extract components and to thermal degradation) is present as well. This leads to a decrease of DPPH radical scavenging activity of the samples.

The general conclusion is that in both cream bases the maximum concentration of the extracts and long storage time do not correspond to the maximum of DPPH scavenging activity of the studied samples. In order to optimize such system parameters as extract concentration and storage time with the aim to obtain a maximum of DPPH radical scavenging activity either more experiments with a varying range of extract concentration or an effective theoretical model which would predict the best system parameters are needed. The former (experimental) approach is very time and sample consuming, since the samples with various extract concentrations should be prepared and stored for 6 weeks. In this work we concentrated on creating an effective theoretical model which would predict the optimum system parameters. This would allow us to reduce the number of experiments to 6 (one per each cream base and extract) and probably also to decrease the time, as the maximum DPPH radical 
scavenging could be reached earlier than after 6 weeks. Once the extract concentration and storage time are predicted with the model, there is no need to perform further measurements. In this work we are going to describe the changes of DPPH radical scavenging activity with time and with concentration for different extracts using ANN.

\subsection{Artificial neural networks}

One of the main advantages of neural networks is their ability to model dependences within the available experimental data with no need of their explicit description. In this work we used ANN to describe interactions between cream base component and berry extract with DPPH radicals as well as the process of oxidation of cream base components. The application of ANN should allow us to predict the DPPH radical scavenging as a function of extract concentration, storage time and cream base, thus the system parameters corresponding to the maximum DPPH radical scavenging activity could be determined.

\subsubsection{ANN modeling}

In this work an MLP type of ANN was used. Generally, the MLP type of ANN with two hidden layers and a sigmoidal activation function was able to spot the trends within the available experimental data (Fig. 1). The developed MLP were trained using different training sets as described above.

Three large training sets consisting of data for each extract (bilberry, elderberry, and aronia) in all cream bases have the advantage that they have more data for analysis. This is always good for ANN training. The drawback is that there are different types of interactions presented in different cream bases, which should be approximated by ANN. So, the ANN trained well on these large training sets, but its performance on previously unseen data was poor (Table I, MLP 2).

In the case of smaller training sets, that contain data for only one type of extract and one type of cream base, the results of ANN training and testing were quite good. The same types of interactions were present in the training set data as in the test set, that is why the ANN performed well on previously unseen data (Table I, MLP 1). Thus, only MLP 1 trained on individual training sets were considered.

As the next step, the developed neural networks were used for the prediction of DPPH scavenging ability of the samples based on the extract type and its concentration, cream base and week of storage in order to enhance the available experimental data. The extract concentration was varied from 0 (pure cream base) to 0.8 and time of storage from 0 to 6 weeks with 1 week step. Based on the obtained results the 3D surface plots were created (Fig. 6). The optimum parameters (extract concentration and time of storage) were determined based on the predicted maximum value of DPPH radical scavenging for each sample (system) (Table II).

As it was shown, the results of ANN prediction are dependent on the training set used for training MLP. Thus, in the case of prediction of pure cream base the DPPH
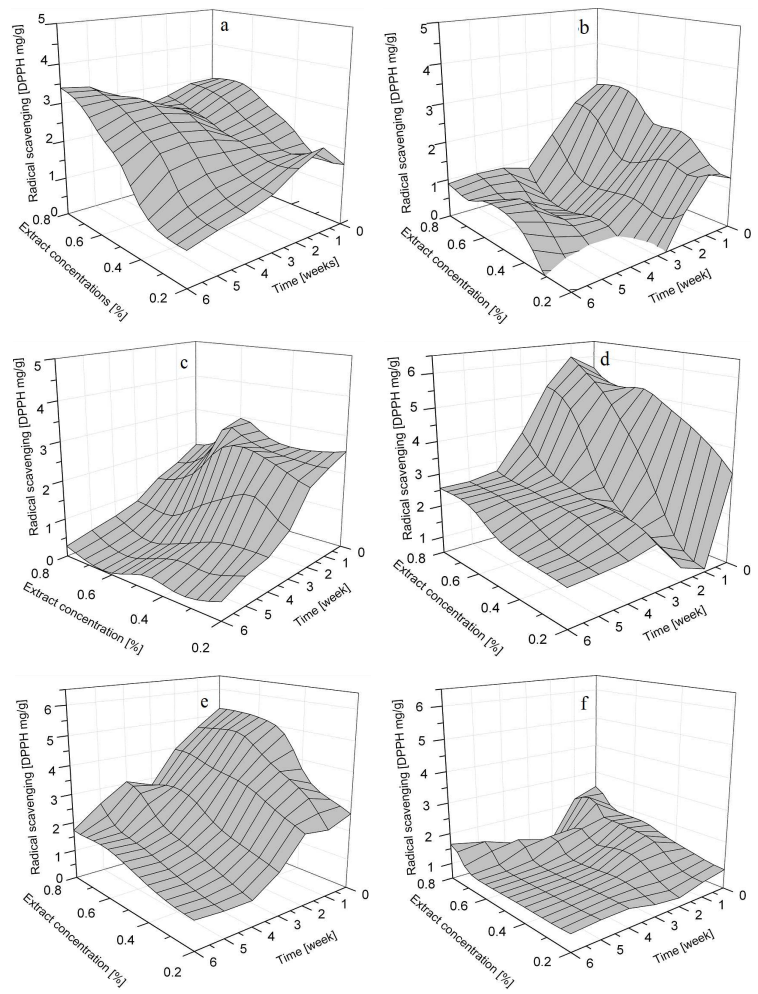

Fig. 6. DPPH radical scavenging $[\mathrm{mg} / \mathrm{g}]$ predicted by ANN - MLP1 (a) aronia extract in w/o base, (b) bilberry extract in $\mathrm{w} / \mathrm{o}$ base, (c) elderberry extract in $\mathrm{w} / \mathrm{o}$ base, (d) aronia extract in $\mathrm{o} / \mathrm{w}$ base, (e) bilberry extract in $\mathrm{o} / \mathrm{w}$ base, (f) elderberry extract in $\mathrm{o} / \mathrm{w}$ base.

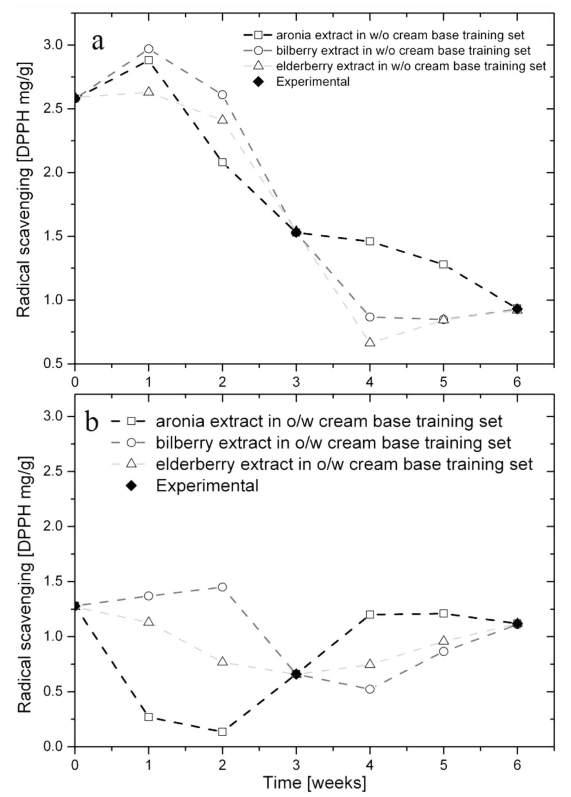

Fig. 7. DPPH radical scavenging of pure cream bases $\mathrm{w} / \mathrm{o}$ (a) and $\mathrm{o} / \mathrm{w}$ (b), experimental results and ANN prediction. ANN was trained using individual training sets, i.e. each training set contains data from one berry extract in one of the cream bases. 
TABLE II

Comparison of the maximum DPPH scavenging activity as predicted by MLP 1 neural network and the corresponding experimental values.

\begin{tabular}{|c|c|c|c|c|c|}
\hline & \multicolumn{3}{|c|}{ Input (max.) } & \multicolumn{2}{|c|}{ Output [mg DPPH $/ \mathrm{g}]$} \\
\hline & $\begin{array}{l}\overline{0} \\
\overrightarrow{3} \\
\dot{3} \\
\dot{0} \\
\dot{0}\end{array}$ & $\underbrace{\frac{y}{d}}$ & $\begin{array}{l}0 \\
\text { జ్ } \\
\tilde{\Xi}\end{array}$ & 萑 & $\overrightarrow{\vec{B}}$ \\
\hline \multirow{2}{*}{ aronia } & 0.8 & 6 & $\mathrm{w} / \mathrm{o}$ & $3.39 \pm 0.16$ & 3.34 \\
\hline & 0.76 & 1 & $\mathrm{o} / \mathrm{w}$ & $2.38 \pm 1.17$ & 5.84 \\
\hline \multirow{2}{*}{ bilberry } & 0.76 & 0 & $\mathrm{w} / \mathrm{o}$ & $2.68 \pm 0.32$ & 2.83 \\
\hline & 0.76 & 0 & $\mathrm{o} / \mathrm{w}$ & $3.63 \pm 0.31$ & 5.26 \\
\hline \multirow{2}{*}{ elderberry } & 0.56 & 1 & $\mathrm{w} / \mathrm{o}$ & $3.9 \pm 0.36$ & 3.01 \\
\hline & 0.76 & 1 & $\mathrm{o} / \mathrm{w}$ & $1.8 \pm 0.39$ & 2.23 \\
\hline $\mathrm{w} / \mathrm{o}$ base & 0 & 1 & $\mathrm{w} / \mathrm{o}$ & $2.93 \pm 0.20$ & 2.97 \\
\hline $\mathrm{o} / \mathrm{w}$ base & 0 & 0 & $\mathrm{o} / \mathrm{w}$ & $1.27 \pm 0.66$ & 1.28 \\
\hline
\end{tabular}

radical scavenging activity obtained from MLP trained on the training sets containing data for only one type of extract and one type of cream base one could expect that the results of prediction would be different. However, irrespectively of the type of extract (and thus, irrespectively of the training set used), all 3 MLP 1 networks are able to predict similar values of $\mathrm{DPPH}$ radical adduct scavenging for w/o cream base (Fig. 7). This means that experimental data for $\mathrm{w} / \mathrm{o}$ cream base used for the construction of training sets for aronia, bilberry, and elderberry underlies the same statistics. It can be concluded that the interaction of $\mathrm{w} / \mathrm{o}$ cream base with DPPH radicals predominates for all samples with w/o cream base, whereas the interaction of the extract and DPPH radicals as well as interactions between the extract and w/o cream base are rather weak. The predominating process includes DPPH scavenging by cream base components and olive oil thermal degradation. This confirms the hypothesis that w/o cream base components have better accessibility to DPPH radicals due to the cream structure. In contrast to $\mathrm{w} / \mathrm{o}$ cream base, the predicted values of the $\mathrm{DPPH}$ radical scavenging activity of $\mathrm{o} / \mathrm{w}$ cream base are strongly dependent on the training set used, and thus, on the type of extract (Fig. 7). These results indicate that interaction of extract and DPPH radicals as well as interaction of extract with cream base components dominate in case of $\mathrm{o} / \mathrm{w}$ cream base. That confirms our suggestion that extracts have better accessibility to DPPH radicals in this cream base.

The above results confirm our assumption that only MLP 1 individually trained for each extract and each cream base could be used for an accurate prediction of $\mathrm{DPPH}$ radical scavenging properties of the samples.

\subsubsection{Creams with berry extracts}

The experimental data extended with the results of ANN prediction give comprehensive information about DPPH scavenging abilities as a function of extract con- centration and storage time (Fig. 7). As it was discussed earlier, the DPPH radical scavenging is determined by a combination of several processes, i.e. the oxidation of olive oil, the changes of cream base structure, and access of the extract to DPPH radicals and cream base components. Based on the results of ANN prediction of DPPH radical scavenging, the samples with bilberry extract have the same trend in DPPH radical scavenging independently of cream base, i.e. the maximum of DPPH radical scavenging is observed after sample preparation using $0.76-0.8 \%$ concentration of berry extract, whereas the minimum of DPPH radical scavenging is predicted for the samples with $0-32 \%$ concentration after just 3 weeks of storage. To conclude, the interaction of cream base components with DPPH radicals predominates in the samples with a low concentration of bilberry extract. At a high concentration of bilberry extract, the additional effect of the interaction of berry extract components with DPPH radicals are observed. Thus, the total $\mathrm{DPPH}$ radical scavenging activity of the samples with bilberry extracts is higher that of the samples of pure cream bases. As expected, the effect of extract components on the increase of DPPH radical scavenging ability is higher in $\mathrm{o} / \mathrm{w}$ cream base $(5.5 \mathrm{mg} \mathrm{DPPH} / 1 \mathrm{~g}$ in freshly prepared samples at $0.76 \%$ extract concentration) than in $\mathrm{w} / \mathrm{o}$ cream base $(3.0 \mathrm{mg} \mathrm{DPPH} / 1 \mathrm{~g}$ in freshly prepared samples at $0.76 \%$ extract concentration) .

In contrast to the samples with bilberry extracts, the predicted DPPH radical scavenging of the samples with aronia and elderberry differ drastically when comparing the results for different cream bases. Thus, ANN predicts that freshly prepared samples with elderberry extract concentration in the range $0-0.6 \%$ in $\mathrm{w} / \mathrm{o}$ cream base will have the highest DPPH radical scavenging. This means that elderberry extract components in w/o cream base have rather weak interactions with DPPH radicals. Moreover, the high concentration of elderberry extract leads to a decrease of DPPH radical scavenging activity. The prooxidant effect of elderberry extract was discussed previously [13]. It was found that the addition of elderberry extract after the beginning of the oxidation reaction leads to the prooxidant effect of the extract. That could be the case in w/o cream base, since the extract components started to interact with the components of cream base (probably with products of olive oil oxidation). In $\mathrm{o} / \mathrm{w}$ cream base, only the antioxidant effect of elderberry extract was observed. This antioxidant effect of elderberry extract was in agreement with previous reports [19].

$\mathrm{DPPH}$ radical scavenging activity of the samples with low concentrations of aronia extract $(0-0.48 \%)$ in w/o cream base are mainly determined by interactions of cream base components with DPPH radicals. High concentrations of aronia extract in freshly prepared samples also result in DPPH radical scavenging activity comparable with that of pure cream base. However, after 6 weeks of storage, a clear effect of extract concentration was observed. In the samples with a low concentration (0- 
$0.12 \%$ ) of aronia extract in $\mathrm{w} / \mathrm{o}$ cream base the DPPH radical scavenging activity decreased, whereas the samples with high concentrations of aronia extracts in w/o cream base after 6 weeks of storage revealed an increase of DPPH radical scavenging activity. This could indicate that in the samples with high concentrations of aronia extract the antioxidant effect of the extract predominates over the process of olive oil oxidation. That is possible when the structure of cream base is changed so that the extract components have better accessibility to DPPH radicals and cream base components.

In contrast to $\mathrm{w} / \mathrm{o}$ cream base, the antioxidant effect of the extract predominates in $\mathrm{o} / \mathrm{w}$ cream base samples with aronia extract. Thus, in freshly prepared samples the DPPH radical scavenging activity increases with the increase of aronia extract concentration. There is an overall decrease of the DPPH radical scavenging activity with time. However, the main trend (an increase of DPPH radical scavenging activity with increase of extract concentration) remains the same.

Based on ANN prediction, the maximum of DPPH radical scavenging was determined for each sample (Table II). Only those samples were prepared and tested. The experimental data are in good agreement with the ANN predicted values. However, in $\mathrm{o} / \mathrm{w}$ cream base the predicted values of DPPH radical scavenging activity of the samples with berry extracts are overestimated as compared with the experimental ones. Probably, the ANN need more experimental results to be trained on, since the underlying processes (interactions of the extracts with cream base components and DPPH radicals) are more sophisticated than those occurring in the samples in $\mathrm{w} / \mathrm{o}$ cream base or in pure $\mathrm{o} / \mathrm{w}$ base.

\section{Conclusion}

The oil-in-water creams with aronia and bilberry extracts show high antioxidant activity as compared with the water-in-oil samples. The highest antioxidant activity was shown for the freshly prepared $0.8 \%$ aronia extract in oil-in-water cream base. Due to various interactions which occur between cream and extract components, the increase of DPPH radical scavenging properties was not always related with the increase of the extract concentration. Moreover, the effect of storage time was different for different berry extracts and cream bases indicating different mechanisms of ageing in those samples. Generally, the oil-in-water cream base appeared to be more stable than water-in-oil, however it was not possible to describe it with the linear model.

The combination of the experimental approach with artificial neural networks provided a comprehensive description of the studied systems. ANN predicted the values of DPPH radical scavenging activity for berry extracts in $\mathrm{w} / \mathrm{o}$ and $\mathrm{o} / \mathrm{w}$ cream bases samples with high accuracy. Thus, a large number of experiments needed to find the optimum concentration and storage time could be safely replaced by ANN modeling. As a result, only 6 additional experiments were performed in order to confirm the results of ANN prediction. The same approach (DPPH-EPR combined with ANN) could be used to optimize the parameters of other herb or berry extract creams and to predict the effect of storage based on the limited number of experiments and samples.

\section{Acknowledgments}

The authors would like to thank Marta Trębińska for performing part of DPPH experiments.

\section{References}

[1] M.C. Branchet, S. Boisnic, C. Frances, A.M. Robert, Gerontology 36, 28 (1990).

[2] Y. Miyachi, J. Dermatol. Sci. 9, 79 (1995).

[3] S. Pillai, C. Oresajo, J. Hayward, Int. J. Cosmetic. Sci. 27, 17 (2005).

[4] S. Briganti, M. Picardo, J. Eur. Acad. Dermatol. Venereol. 17, 663 (2003).

[5] D. Darr, S. Combs, S. Dunston, T. Manning, S. Pinnell, Br. J. Dermatol. 127, 247 (1992).

[6] F. Dreher, B. Gabard, D.A. Schwindt, H.I. Maibach, Br. J. Dermatol. 139, 332 (1998).

[7] J.C. Murray, J.A. Burch, R.D. Streilein, M.A. Iannacchione, R.P. Hall, S.R. Pinnell, J. Am. Acad. Dermatol. 59, 418 (2008).

[8] A. Chaovanalikit, R.E. Wrolstad, J. Food Sci. 69 FCT67 (2004).

[9] H. Wang, G. Cao, R.L. Prior, J. Agric. Food Chem. 44, 701 (1996).

[10] R.L. Prior, G. Cao, A. Martin, E. Sofic, J. McEwen, C. O'Brien, N. Lischner, M. Ehlenfeldt, W. Kalt, G. Krewer, C.M. Mainland, J. Agric. Food Chem. 46, 2686 (1998).

[11] D. Bagchi, C.K. Sen, M. Bagchi, M. Atalay, Biochemistry (Moscow) 69, 75 (2004).

[12] M.P. Kähkönen, A.I. Hopia, M. Heinonen, J. Agric. Food Chem. 49, 4076 (2001).

[13] P.M. Abuja, M. Murkovic, W. Pfannhauser, J. Agric. Food Chem. 46, 4091 (1998).

[14] W. Kalt, J.E. McDonald, R.D. Ricker, X. Lu, Can. J. Plant Sci. 79, 617 (1999).

[15] R.L. Prior, G. Cao, A. Martin, E. Sofic, J. McEwen, C. O'Brien, L. Lischner, M. Ehlenfeldt, W. Kalt, G. Krewer, C.M. Mainland, J. Agric. Food Chem. 46, 2686 (1998).

[16] J. Oszmianski, J.C. Sapis, J. Food Sci. 53, 1241 (1988).

[17] G. Mazza, E. Miniati, Anthocyanins in Fruits, Vegetables and Grains, CRC Press, Boca Raton 1993.

[18] R.A. Moyer, K.E. Hummer, C.E. Finn, B. Frei, R.E. Wrolstad, J. Agric. Food Chem. 50, 519 (2002).

[19] L. Jakobek, M. Šeruga, I. Novak, M.F. MedvidovićKosanović, Deut. Lebensm.-Rundsch 103, 369 (2007).

[20] M.J. Bermúdez-Soto, F.A. Tomás-Barberán, Eur. Food Res. Technol. 219, 133 (2004). 
[21] M. Ozgen, J.C. Scheerens, R.N. Reese, R.A. Miller, Pharmacogn. Mag. 6, 198 (2010).

[22] D. Burdulis, A. Šarkinas, I. Jasutiené, E. Stackevičené, L. Nikolajevas, V. Janulis, Acta Pol. Pharm. 66, 399 (2009).

[23] M.P. Kähkönen, J. Heinämäki, V. Ollilainen, M. Heinonen, J. Sci. Food Agric. 83, 1403 (2003).

[24] J. Oszmiański, A. Wojdylo, Eur. Food Res. Technol. 221, 809 (2005).

[25] S. Valcheva-Kuzmanova, B. Blagović, S. Valić, Pharmacogn. Mag. 8, 171 (2012).

[26] Q. Guo, B. Zhao, S. Shen, J. Hou, J. Hu, W. Xin, Biochim. Biophys. Acta (BBA) - Gen. Subjects 1427, 13 (1999).

[27] W.-C. Hou, F.-L. Hsu, M.-H. Lee, Planta Med. 68, $1072(2002)$

[28] D. Sanna, G. Delogu, M. Mulas, M. Schirra, A. Fadda, Food Anal. Meth. 5, 759 (2012).
[29] M. Bartoszek, J. Polak, Food Chem. 132, 2089 (2012).

[30] M. Zalibera, A. Staško, A. Šlebodová, V. Jančovičová, T. Čermáková, V. Brezová, Food Chem. 110, 512 (2008).

[31] M.C. Meinke, F. Syring, S. Schanzer, S.F. Haag, R. Graf, M. Loch, I. Gersonde, N. Groth, F. Pflücker, J. Lademann, Photochem. Photobiol. 89, 1079 (2013).

[32] A. Valanidis, C. Nisiotou, Y. Papageorgiou, I. Kremli, N. Satravelas, N. Zinieris, H. Zygalaki, J. Agric. Food Chem. 52, 2358 (2004).

[33] S. Gasic, B. Jovanovic, S. Jovanovic, J. Serb. Chem. Soc. 67, 31 (2002). 